\title{
EEG correlates associated with the severity of gambling disorder and serum BDNF levels in patients with gambling disorder
}

\author{
KYOUNG MIN KIM ${ }^{1}$, SAM-WOOK CHOI ${ }^{2}$, JAEWON LEE $^{3 *}$ and JUN WON KIM ${ }^{4} *$ \\ ${ }^{1}$ Department of Psychiatry, Dankook University Hospital, Cheonan, Republic of Korea \\ ${ }^{2}$ Department of Psychiatry, Korea Institute on Behavioral Addictions, True Mind Mental Health Clinic, Seoul, Republic of Korea \\ ${ }^{3}$ Department of Psychiatry, Korea Institute on Neuromodulation, Easybrain Center, Seoul, Republic of Korea \\ ${ }^{4}$ Department of Psychiatry, Catholic University of Daegu School of Medicine, Daegu, Republic of Korea
}

(Received: December 21, 2017; revised manuscript received: April 8, 2018; accepted: April 14, 2018)

\begin{abstract}
Background and aims: This study aimed to evaluate the association between the severity of pathological gambling, serum brain-derived neurotrophic factor (BDNF) level, and the characteristics of quantitative electroencephalography (EEG) in patients with gambling disorder. Methods: A total of 55 male patients aged 18-65 with gambling disorder participated. The severity of pathological gambling was assessed with the nine-item Problem Gambling Severity Index from the Canadian Problem Gambling Index (CPGI-PGSI). The Beck Depression Inventory and Lubben Social Network Scale were also assessed. Serum BDNF levels were assessed from blood samples. The resting-state EEG was recorded while the eyes were closed, and the absolute power of five frequency bands was analyzed: delta (1-4 Hz), theta $(4-8 \mathrm{~Hz})$, alpha $(8-12 \mathrm{~Hz})$, beta $(12-30 \mathrm{~Hz})$, and gamma $(30-50 \mathrm{~Hz})$. Results: Serum BDNF level was positively correlated with theta power in the right parietal region $(\mathrm{P} 4, r=.403, p=.011)$, beta power in the right parietal region $(\mathrm{P} 4, r=.456, p=.010)$, and beta power in the right temporal region (T8, $r=.421, p=.008)$. Gambling severity (CPGI-PGSI) was positively correlated with absolute beta power in the left frontal region (F7, $r=.284$, $p=.043)$ and central region $[(\mathrm{C} 3, r=.292, p=.038),(\mathrm{C} 4, r=.304, p=.030)]$. Conclusions: These findings support the hypothesis that right-dominant lateralized correlations between BDNF and beta and theta power reflect rightdominant brain activation in addiction. The positive correlations between beta power and the severity of gambling disorder may be associated with hyperexcitability and increased cravings. These findings contribute to a better understanding of brain-based electrophysiological changes and BDNF levels in patients with pathological gambling.
\end{abstract}

Keywords: quantitative electroencephalography, resting state, BDNF, gambling disorder

\section{INTRODUCTION}

Gambling is a popular leisure enjoyed by $60 \%-90 \%$ of people at least once in their lives, pathological gambling results in health, financial, and social problems (Wölfling et al., 2011). Gambling disorder is defined by persistent and recurrent maladaptive gambling behavior that disrupts personal, family, and vocational pursuits (American Psychiatric Association [APA], 2013). The lifetime prevalence of gambling disorder is $0.4 \%-1.0 \%$ (APA, 2013). Gambling disorder is known to be highly comorbid with other psychiatric disorders, such as substance-use disorder, depressive disorder, and anxiety disorder (APA, 2013; Lorains, Cowlishaw, \& Thomas, 2011).

Brain-derived neurotrophic factor (BDNF) is an important regulator of neural survival, development, function, and plasticity (Huang \& Reichardt, 2001). BDNF is highly expressed in limbic structures and the cerebral cortex, and plays an important role in learning, memory, and rewardrelated processes (Boulle et al., 2012; Yamada \& Nabeshima, 2003). It is associated with multiple mental disorders, including depression, anxiety, schizophrenia, and addiction (Boulle et al., 2012; Li \& Wolf, 2015). Several studies have reported that BDNF is elevated in patients with pathological gambling (Angelucci et al., 2013; Choi et al., 2016; Geisel, Panneck, Hellweg, Wiedemann, \& Müller, 2015). Researchers have suggested that the increased BDNF in pathological gamblers is related to altered dopaminergic transmission in the ventral tegmental area and nucleus accumbens, which are central components of the brain's reward system (Geisel, Banas, Hellweg, \& Müller, 2012; $\mathrm{Pu}$, Liu, \& Poo, 2006).

Quantitative electroencephalography (qEEG) involves power spectral analysis of frequency bands, such as delta

\footnotetext{
* Corresponding authors: Jaewon Lee, MD, PhD; Department of Psychiatry, Korea Institute on Neuromodulation, EasyBrain Center, 1330-9 Seocho-dong, Seocho-gu, Seoul, Republic of Korea; Phone: +82 2583 9081; Fax: +82 2583 9082; E-mail: sonton21@gmail.com; Jun Won Kim, MD, PhD; Department of Psychiatry, Catholic University of Daegu School of Medicine, 33 Duryugongwon-ro 17-gil, Nam-Gu, Daegu 42472, Republic of Korea; Phone: +82 53650 4332; Fax: +82 53623 1694; E-mail: f_affection@naver.com
}

This is an open-access article distributed under the terms of the Creative Commons Attribution-NonCommercial 4.0 International License, which permits unrestricted use, distribution, and reproduction in any medium for non-commercial purposes, provided the original author and source are credited, a link to the CC License is provided, and changes - if any - are indicated. 
(1-4 Hz), theta $(5-7 \mathrm{~Hz})$, alpha $(8-13 \mathrm{~Hz})$, and beta (14-30 Hz) (Houston \& Ceballos, 2013). Pathological gamblers show dysfunctional EEG activity, especially in the frontoparietal area (Quintero, 2017). Some studies of the EEG correlates of gambling behavior have focused on reward sensitivity and decision-making (Houston \& Ceballos, 2013). For example, theta and delta activities are correlated with various aspects of the decision-making process (Houston \& Ceballos, 2013). Massar, Rossi, Schutter, and Kenemans (2012) reported that an increased theta-beta ratio in the resting-state EEG to be associated with risky or disadvantageous decision-making in the Iowa Gambling Task. Amoss (2009) reported that beta power asymmetry was associated with the number of risky decision-making behaviors.

Gambling disorder is closely related to impulsivity, which is a core feature of addictive disorders. In spite of some inconsistent findings (Lee et al., 2017), studies of EEG correlates in impulsive disorders, such as attention-deficit/ hyperactivity disorder, substance-use disorder, and violence, reported relatively consistent findings of increases in the delta, theta, and beta bands (Kamarajan \& Porjesz, 2012). Increased power of slow waves, such as the delta and theta bands, suggests low cortical arousal, whereas increased power in the beta band suggests hyperexcitability of the central nervous system.

A relationship between BDNF and qEEG has been described in patients with depressive disorder (Gatt et al., 2008; Zoon et al., 2013). However, there has been no study of the association between BDNF and qEEG power specifically in patients with gambling disorder. Thus, this study aimed to investigate the associations between serum BDNF level, qEEG power distribution, and the severity of pathological gambling in patients with gambling disorder.

\section{METHODS}

\section{Subjects}

Individuals who visited the gambling disorder clinic were considered for inclusion in the study. The inclusion criteria were a diagnosis of gambling disorder, according to the fifth edition of the Diagnostic and Statistical Manual of Mental Disorders (DSM-5) criteria, male sex, and age between 18 and 65 years. Subjects were excluded if any of the following applied: a diagnosis of substance-use disorder other than nicotine or caffeine, based on the DSM-5 criteria; use of psychotropic medications over the previous year; and presence of a physical, mental, or neurological disorder other than gambling disorder. Based on the inclusion and exclusion criteria, 55 male subjects (age: $38.42 \pm 11.59$ years) were enrolled in the study. None of the participants were taken any medications and all had completed at least 12 years of education (mean: $14.65 \pm 1.89$ years).

\section{Measures}

To assess serum BDNF level, a total of $10 \mathrm{ml}$ of blood was drawn from each subject and placed in a serum separator tube. For analysis of serum, samples were allowed to clot for $30 \mathrm{~min}$ at room temperature before centrifugation for $15 \mathrm{~min}$ at approximately $1,000 \times g$, after which the serum was removed. All samples were stored at $-80{ }^{\circ} \mathrm{C}$. Serum BDNF levels were determined using an enzyme-linked immunosorbent assay protocol according to the manufacturer's instructions (DBD00, R\&D Systems, Europe). The BDNF level values were normally distributed, as verified by the Kolmogorov-Smirnov test.

The nine-item Problem Gambling Severity Index (PGSI) from the Canadian Problem Gambling Index (CPGI) (CPGIPGSI) was selected to quantify gambling severity (Ferris \& Wynne, 2001). The PGSI was used to assess problematic gambling behavior and adverse consequences during the previous 12 months. The response choices for each PGSI item are "never," "sometimes," "most of the time," and "almost always," with the total score ranging from 0 to 27 . Participants were categorized as "non-problem-gamblers" $(\mathrm{PGSI}=0)$, "low-risk" (PGSI = 1-2), "moderate-risk" (PGSI = 3-7), or "problem-gambler" (PGSI > 7). The psychometric properties of the PGSI have been examined in the Korean population; Cronbach's $\alpha$ value was 94 (Kim, Cha, Kwon, \& Lee, 2011). Beck Depression Inventory (BDI) and Lubben Social Network Scale were also used to assess the depressive mood and social activity of the participants.

\section{EEG recording and preprocessing}

The EEG recordings were performed using a SynAmps2 direct-current (DC) amplifier and a 10-20 layout 64-channel Quick-Cap electrode-placement system (Neuroscan Inc., NC, USA). The EEG data were digitally recorded from 19 gold cup electrodes placed according to the international 10-20 system. The impedances were maintained below $5 \mathrm{k} \Omega$, and the sampling rate was $1,000 \mathrm{~Hz}$. We used a linked-mastoid reference and two additional bipolar electrodes to measure horizontal and vertical eye movements. During the recordings, each participant laid down in a semidarkened, electrically shielded, sound-attenuated room. Resting EEG samples were recorded after 3 min with the participant's eyes closed.

We used Matlab 7.0.1 (Math Works, Natick, MA, USA) and the EEGLAB toolbox (Delorme \& Makeig, 2004) to preprocess and analyze the EEG recordings. First, the EEG data were downsampled to $250 \mathrm{~Hz}$. Next, the EEG data were detrended and mean-subtracted to remove the DC component. A 1-Hz high-pass filter and a $60-\mathrm{Hz}$ notch filter were applied to remove eye movement artifacts and electrical noise. Independent component analysis (ICA) was then performed to remove well-defined sources of artifacts. ICA has been demonstrated to reliably isolate artifacts caused by eye and muscle movements and heart noise (Jung et al., 2000). Finally, clinical psychiatrists and EEG experts visually inspected the corrected EEGs. For the analysis, we selected at least $2 \mathrm{~min}$ of artifact-free EEG data from the 3 -min recordings.

\section{EEG analysis}

Four frequency bands were defined for further analysis: delta $(1-4 \mathrm{~Hz})$, theta $(4-8 \mathrm{~Hz})$, alpha $(8-12 \mathrm{~Hz})$, and beta $(12-30 \mathrm{~Hz})$. We investigated the power spectra of the EEG data from each subject using the short-time-interval Fourier 
transform "spectrogram.m" function from the Signal Processing Toolbox in MATLAB. Time windows of 1,000 ms with an 800-ms overlap and Hamming window were used for the spectral analysis. Outliers that were far from the spectral value distribution for each frequency band, at the 0.05 significance level, were removed. Finally, the absolute power for each frequency band was averaged over all the time windows for further analysis.

\section{Statistical analysis}

The MATLAB 7.0.1 Statistical Toolbox was used for the statistical analyses. All values were expressed as the mean and standard deviation $(S D)$. To assess the relationship between gambling severity (CPGI-PGSI) and EEG recordings, we used a Pearson's partial correlation analysis that controlled for age, education, BDI score, and Beck Anxiety Inventory (BAI) score. To assess the relationship between BDNF and the EEG recordings, we used the same analytic method. Statistical significance was defined as $p<.05$. To control for false positives from multiple comparisons, we used a false discovery rate (FDR) correction in which the $p$ values were multiplied by the number of comparisons (Benjamini \& Hochberg, 1995).

\section{Ethics}

The study procedure was performed in accordance with the Declaration of Helsinki (World Medical Association, 1964). The study protocols were approved by the Regional Ethical Review Board in Seoul. All participants gave written informed consent after receiving a complete description of the study and were not compensated for taking part in the study.

\section{RESULTS}

\section{Demographic and clinical characteristics}

The total sample comprised 55 male individuals with gambling disorder [mean age $(S D)=38.42$ (11.59) years]. The participants showed clinically higher scores compared with the general population on the BDI [mean $(S D)=16.64$ (9.42)] and CPGI-PGIS [mean $(S D)=18.87$ (6.18)] (Kim et al., 2011; Lasa, Ayuso-Mateos, Vazquez-Barquero, Diez-Manrique, \& Dowrick, 2000). Their scores on the Lubben Social Network Scale [mean $(S D)=24.71$ (5.35)] were low level and reflected a limited social network (Lee et al., 2009). The demographic and clinical characteristics are summarized in Table 1.

\section{Correlation between serum BDNF level and absolute power} of resting EEG

To investigate whether the serum BDNF levels were related to the absolute power of the resting EEG, we examined partial correlations corrected for age, education, BDI, and BAI. The partial correlation analysis for the absolute delta and alpha power and the serum BDNF levels showed no significant correlations, after imposing the FDR
Table 1. Demographic and clinical characteristics of subjects

\begin{tabular}{lc}
\hline Mean $\pm S D$ & GD $(n=55)$ \\
\hline Age (years) & $38.42 \pm 11.59$ \\
Education (years) & $14.65 \pm 1.89$ \\
BDNF (pg/ml) & $32,177.69 \pm 9,969.95$ \\
BDI & $16.64 \pm 9.42$ \\
BAI & $12.58 \pm 9.68$ \\
BIS & $56.42 \pm 8.28$ \\
LSNS & $24.71 \pm 5.35$ \\
WURS & $30.07 \pm 15.80$ \\
CPGI-PGIS & $18.87 \pm 6.18$ \\
RTCQ & \\
Precontemplation & $2.09 \pm 2.47$ \\
Contemplation & $5.67 \pm 2.04$ \\
Action & $4.23 \pm 2.58$ \\
\hline
\end{tabular}

Note. SD: standard deviation; GD: gambling disorder; BDNF: brain-derived neurotrophic factor; BDI: Beck Depression Inventory; BAI: Beck Anxiety Inventory; BIS: The Korean version of Barratt Impulsiveness Scale; LSNS: Lubben Social Network Scale; WURS: Wender-Utah Rating Scale; CPGI-PGIS: Canadian Problem Gambling Index-Problem Gambling Severity Index; RTCQ: Readiness To Change Questionnaire.

correction. The serum BDNF level was positively correlated with theta power in the right parietal region (P4, $r=.403, p=.011)$, beta power in the right parietal region ( $\mathrm{P} 4, r=.456, p=.010)$, and beta power in the right temporal region $(\mathrm{T} 8, r=.421, p=.008)$. All these significance assessments include FDR corrections (corrected $p<.05)$. A topographical representation of the Pearson's partial correlation coefficients and the associated $p$ values is presented in Figure 1.

\section{Correlation between gambling severity and absolute power} of resting EEG

The partial correlation analysis, controlled for age, education, BDI, and BAI, for the absolute delta, theta, and alpha power and gambling severity (CPGI-PGSI) showed no significant relationships. However, gambling severity (CPGI-PGSI) was positively correlated with absolute beta power. The significant findings appeared in the left frontal region $(\mathrm{F} 7, r=.284, p=.043)$ and central region $[(\mathrm{C} 3, r=.292, p=.038),(\mathrm{C} 4, r=.304, p=.030)]$. All significant differences were FDR-corrected (corrected $p<.05)$. Scatter plots of the three significant $(p<.01)$ partial correlations are presented in Figure 2.

\section{DISCUSSION}

This study investigated associations between the severity of pathological gambling, serum BDNF level, and the characteristics of qEEG power in patients with gambling disorder. Several previous studies have reported an association between BDNF and qEEG power. Gatt et al. (2008) reported that the $B D N F$ Val66Met gene polymorphism was associated with EEG power in depressive patients: carriers of the methionine (Met) variant, who show phenotypic 

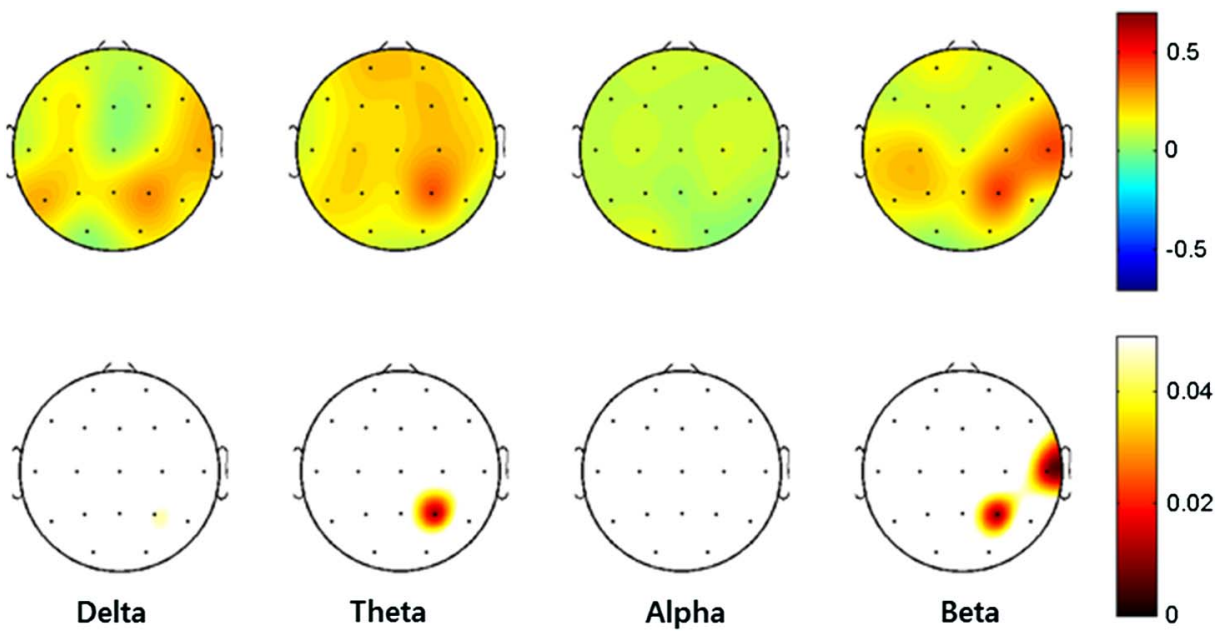

Figure 1. Topographical representations of the Pearson's partial correlations, corrected for age, education, BDI, and BAI, between the absolute powers and the serum BDNF levels. Scales show uV2 for absolute power. Red represents higher values and blue represents lower values. The upper topography denotes the Pearson's partial correlation coefficients, and the lower topography denotes the $p$ values after adjusting for the false discovery rate (corrected $p<.05$ ). BDI: Beck Depression Inventory; BAI: Beck Anxiety Inventory; BDNF: brain-derived neurotrophic factor
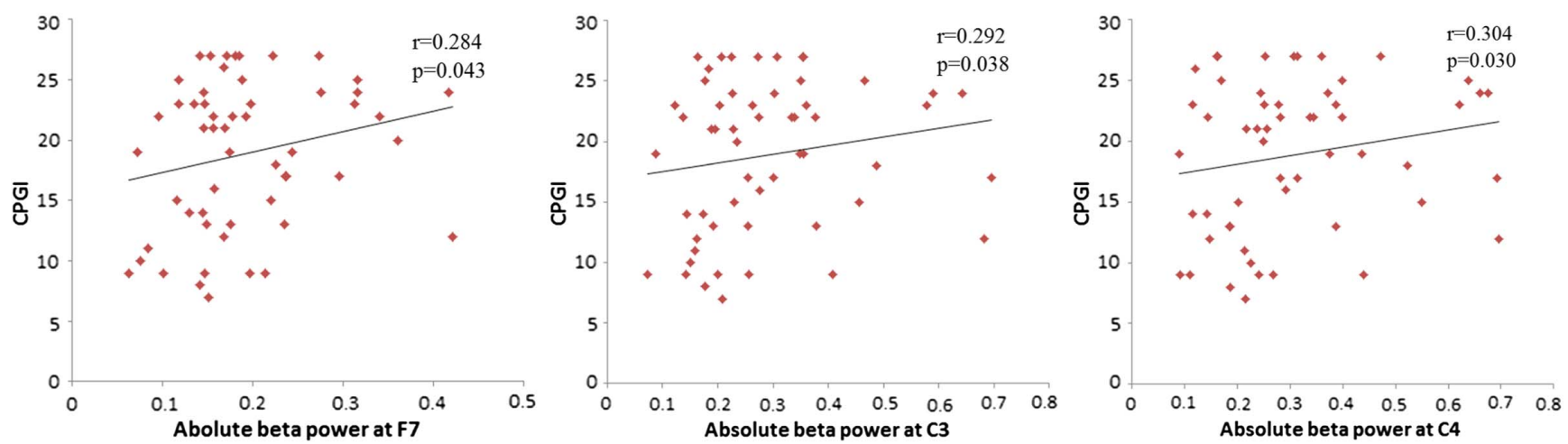

Figure 2. Correlation of the absolute beta power at the F7, C3, and C4 electrodes site with scores on the Canadian Problem Gambling Index (CPGI) in patients with gambling disorder

characteristics of depression, presented elevated relative theta and delta power coupled with reduced alpha power compared to carriers of the valine (Val) variant. Zoon et al. (2013) also reported that parietal-occipital alpha power was negatively associated with depression severity in depressive patients, and those Met/Met depressive patients showed lower global absolute alpha power compared to Val carriers.

However, those studies of the association between BDNF and $\mathrm{qEEG}$ were performed with depressive patients. In addition, those studies investigated the association between EEG power and $B D N F$ gene polymorphism. Notably, a meta-analysis indicated that the $B D N F$ Val66Met variant is not associated with serum BDNF level (Terracciano et al., 2013). This is the first study of the association between BDNF and qEEG power in patients with gambling disorder. Furthermore, we investigated the associations of qEEG band power and BDNF in terms of the serum BDNF level, not in terms of $B D N F$ gene polymorphism. We identified significant positive correlations between serum BDNF level and beta and theta power in the right parietal and temporal regions of the gambling disorder patients.
BDNF is known to be involved in learning and memory, which are the functions of the hippocampus (Yamada \& Nabeshima, 2003). Some researchers have reported that the $B D N F$ Val66Met polymorphism is associated with the volume of the hippocampal formation (Pezawas et al., 2004; Szeszko et al., 2005). Grunwald, Hensel, Wolf, Weiss, and Gertz (2007) reported that an increase in EEG theta power was associated with decreased hippocampal volume. Thus, it is plausible that the correlations between BDNF and EEG power in the temporal and parietal lobes, which were identified in this study, might be associated with the anatomical location of the hippocampus. However, this explanation requires confirmation by further studies using MRI data.

With regard to the EEG asymmetry, multiple studies have reported a right-dominant resting frontal EEG asymmetry in alpha power in relation to depression and anxiety (Thibodeau, Jorgensen, \& Kim, 2006). Bulgin et al. (2008) reported that theta EEG asymmetry in parietal brain regions was affected by the $B D N F$ Val66Met polymorphism in patients with childhood-onset mood disorder. In a functional MRI study of the laterality of brain activation in addictive disorders, Gordon (2016) reported right dominance of brain 
activation for impulsivity tasks and left dominance for cueinduced craving in most addictive disorders, including addictions to cocaine, heroin, and food. However, cueinduced craving for Internet gaming, which is a behavioral addiction comparable to gambling, showed right-dominated brain activation. The right-favoring lateralized correlations between BDNF and beta and theta power identified in this study reflect consistent findings, which should be confirmed by further studies.

Another main finding of this study is the positive correlation between beta activity and the severity of pathological gambling, as measured by the CPGI-PGSI. The highfrequency bands (beta and gamma) are involved in local, short-range neural communication (Von Stein \& Sarnthein, 2000), and increased beta power is considered to indicate hyperexcitability in the brain (Rangaswamy et al., 2002). Several psychiatric disorders characterized by impulsivity and hyperarousal, including intermittent explosive disorder and substance-use disorders, were reported to be associated with increased beta activity (Kamarajan \& Porjesz, 2012). Herrera-Díaz et al. (2016) also reported increased absolute and relative beta power in alcohol-use disorder patients compared to controls.

Other researchers have focused on associations between craving and beta activity. Knott et al. (2008) reported that a scripted scene eliciting the desire to smoke-produced craving response and increased beta power in smokers. Arras (2017) reported that women with uncontrollable eating show increased reward sensitivity, which is correlated with elevated beta activity during the resting state. Tammela et al. (2010) reported that women with binge-eating showed higher resting-state beta power than women without binge-eating, and that pictures of food increased beta power in women both with and without binge-eating. Increased high beta power in response to positive feedback was also reported to be associated with activation in a largely subcortical network encompassing core areas of the reward network (Andreou et al., 2017).

It is plausible that the positive correlations between beta power and CPGI-PGSI identified in this study may be associated with hyperexcitability and increased craving in people with gambling disorders. Further studies are required to confirm these associations, including studies using other physiologic indices or experimental procedures to assess hyperexcitability and craving.

Interestingly, previous studies of Internet addiction, which is a behavioral addiction comparable to gambling disorder, have reported some inconsistent findings. An Internet gaming disorder group was reported to show lower absolute beta power than healthy control groups (Son et al., 2015). However, Park et al. (2017) reported that an Internet gaming disorder group showed increased gamma band (30-40 Hz) coherence compared to an alcohol-use disorder group and healthy controls. Choi et al. (2013) also reported that an Internet addiction group showed higher absolute power in the gamma band than controls, whereas the beta power of the Internet addiction group was lower than that of the controls. These reports may be consistent with the finding that increased activity in the higher frequency bands is associated with pathological hyperexcitability in behavioral addictions.
Notably, previous studies of serum BDNF levels in patients with gambling disorder have reported inconsistent findings. On one hand, the serum BDNF level has been reported to increase in patients with gambling disorder compared to healthy control (Choi et al., 2016; Geisel et al., 2012). On the other hand, although Choi et al. (2016) also reported a positive correlation between serum BDNF level and the severity of the gambling disorder, there was no significant correlation between BDNF serum level and the severity of pathological gambling in the study of Geisel et al. (2012). This study also did not find a significant correlation between serum BDNF level and the severity of pathological gambling.

\section{Limitations}

This study has several limitations that should be noted. First, this study did not include healthy controls and therefore does not allow comparisons between the patients and controls. A future case-control design is required to confirm our findings. Second, the age range of the participants was broad, that is, 18-65. The power of EEG frequency bands is known to vary according to age, although the changes in EEG power during adulthood are less remarkable than those in childhood (Dustman, Shearer, \& Emmerson, 1999). The characteristics of gambling can also show age-dependent features (Kardos, Tóth, Boha, File, \& Molnár, 2017). A future study with an age-matching design or narrow age span is warranted to overcome this limitation. Third, the severity of gambling was assessed by self-administered questionnaires alone. In addition, we did not collect information on the clinical history of gambling. Patients with gambling disorder often have poor insight, so more objective tools for the assessment of gambling severity and a thorough clinical history of the patients are required. Fourth, although qEEG could be affected by handedness, and studies for qEEG usually consider the handedness of the participants, we did not take handedness into account. However, only $4.2 \%$ of Koreans were reported to be lefthanded in a study of Korean college students (Kang \& Harris, 2000). Thus, the effect of handedness on the results of this study is expected to be minimal. Finally, only 19 electrodes were used in this study, which is not enough to evaluate topographical functions. To resolve topographical issues, EEG source reconstruction is required, but is not possible with the relatively small number of 19 channels. To compensate this limitation, previous studies have combined EEG with MRI or used more electrodes (Lei, Wang, Yuan, \& Mantini, 2014; Mantini, Perrucci, Del Gratta, Romani, \& Corbetta, 2007). Therefore, a future study with more electrodes is warranted.

\section{CONCLUSIONS}

This study extends the understanding of the associations between serum BDNF level, EEG characteristics in the resting state, and the severity of gambling disorder. The results showed a positive correlation between serum BDNF level and theta and beta power in the right temporoparietal region, and a positive correlation between the severity of 
pathological gambling and beta power in the frontal and central regions. The right-dominant lateralized correlations between BDNF and beta and theta power might reflect the right-dominant brain activation that is reported in most addictive disorders. The positive correlations between beta power and the severity of gambling disorder may be associated with hyperexcitability and increased cravings. These findings suggest the possibility of objective measurement of the severity of gambling disorder and neurophysiological state of the patients, and may lead to useful clinical information in the form of neurobiological markers for assessment and treatment planning for patients with gambling disorder.

Funding sources: This work was supported by a grant from the Korea Healthcare Technology R\&D Project, Ministry for Health and Welfare, South Korea (no. A120157).

Authors' contribution: All authors are accessible to the study data and responsible for preparing all contents of the article. All authors had authority over the decision to submit the manuscript for publication. KMK and S-WC contributed equally to this work.

Conflict of interest: The authors declare no conflict of interest.

\section{REFERENCES}

Amoss, R. T. (2009). Frontal alpha and beta EEG power asymmetry and Iowa Gambling Task performance. Atlanta, GA: Georgia State University.

Andreou, C., Frielinghaus, H., Rauh, J., Mußmann, M., Vauth, S., Braun, P., Leicht, G., \& Mulert, C. (2017). Theta and high-beta networks for feedback processing: A simultaneous EEG-fMRI study in healthy male subjects. Translational Psychiatry, 7(1), e1016. doi:10.1038/tp.2016.287

Angelucci, F., Martinotti, G., Gelfo, F., Righino, E., Conte, G., Caltagirone, C., Bria, P., \& Ricci, V. (2013). Enhanced BDNF serum levels in patients with severe pathological gambling. Addiction Biology, 18(4), 749-751. doi:10.1111/j.1369-1600. 2011.00411.x

Arras, L. (2017). Reward sensitivity and self-control in uncontrolled eating: Analysis of EEG beta and theta dynamics. Tartu, Estonia: University of Tartu.

American Psychiatric Association [APA]. (2013). Diagnostic and statistical manual of mental disorders (DSM-5). Arlington, VA: American Psychiatric Association.

Benjamini, Y., \& Hochberg, Y. (1995). Controlling the false discovery rate: A practical and powerful approach to multiple testing. Journal of the Royal Statistical Society. Series B (Methodological), 57(1), 289-300. doi:10.2307/2346101

Boulle, F., van den Hove, D. L., Jakob, S. B., Rutten, B. P., Hamon, M., van Os, J., Lesch, K. P., Lanfumey, L., Steinbusch, H. W., \& Kenis, G. (2012). Epigenetic regulation of the BDNF gene: Implications for psychiatric disorders. Molecular Psychiatry, 17(6), 584-596. doi:10.1038/mp.2011.107

Bulgin, N. L., Strauss, J. S., King, N. A., Shaikh, S. A., George, C. J., Fox, N. A., Barr, C. L., Kovacs, M., \& Kennedy, J. L. (2008). Association study of theta EEG asymmetry and brainderived neurotrophic factor gene variants in childhood-onset mood disorder. Neuromolecular Medicine, 10(4), 343-355. doi:10.1007/s12017-008-8038-x

Choi, J.-S., Park, S. M., Lee, J., Hwang, J. Y., Jung, H. Y., Choi, S.-W., Oh, S., \& Lee, J.-Y. (2013). Resting-state beta and gamma activity in Internet addiction. International Journal of Psychophysiology, 89(3), 328-333. doi:10.1016/j.jpsycho.2013.06.007

Choi, S.-W., Shin, Y.-C., Mok, J. Y., Kim, D.-J., Choi, J.-S., \& Suk-Hyun Hwang, S. (2016). Serum BDNF levels in patients with gambling disorder are associated with the severity of gambling disorder and Iowa Gambling Task indices. Journal of Behavioral Addictions, 5(1), 135-139. doi:10.1556/2006.5. 2016.010

Delorme, A., \& Makeig, S. (2004). EEGLAB: An open source toolbox for analysis of single-trial EEG dynamics including independent component analysis. Journal of Neuroscience Methods, 134(1), 9-21. doi:10.1016/j.jneumeth.2003.10.009

Dustman, R., Shearer, D., \& Emmerson, R. (1999). Life-span changes in EEG spectral amplitude, amplitude variability and mean frequency. Clinical Neurophysiology, 110(8), 1399-1409. doi:10.1016/S1388-2457(99)00102-9

Ferris, J. A., \& Wynne, H. J. (2001). The Canadian Problem Gambling Index. Ottawa, ON: Canadian Centre on Substance Abuse.

Gatt, J. M., Kuan, S. A., Dobson-Stone, C., Paul, R. H., Joffe, R. T., Kemp, A. H., Gordon, E., Schofield, P. R., \& Williams, L. M. (2008). Association between BDNF Val66Met polymorphism and trait depression is mediated via resting EEG alpha band activity. Biological Psychology, 79(2), 275-284. doi:10.1016/j.biopsycho.2008.07.004

Geisel, O., Banas, R., Hellweg, R., \& Müller, C. A. (2012). Altered serum levels of brain-derived neurotrophic factor in patients with pathological gambling. European Addiction Research, 18(6), 297-301. doi:10.1159/000338281

Geisel, O., Panneck, P., Hellweg, R., Wiedemann, K., \& Müller, C. A. (2015). Hypothalamic-pituitary-adrenal axis activity in patients with pathological gambling and Internet use disorder. Psychiatry Research, 226(1), 97-102. doi:10.1016/j.psychres. 2014.11.078

Gordon, H. W. (2016). Laterality of brain activation for risk factors of addiction. Current Drug Abuse Reviews, 9(1), 1-18. doi:10.2174/1874473709666151217121309

Grunwald, M., Hensel, A., Wolf, H., Weiss, T., \& Gertz, H.-J. (2007). Does the hippocampal atrophy correlate with the cortical theta power in elderly subjects with a range of cognitive impairment? Journal of Clinical Neurophysiology, 24(1), 22-26. doi:10.1097/WNP.0b013e31802ed5b2

Herrera-Díaz, A., Mendoza-Quiñones, R., Melie-Garcia, L., Martínez-Montes, E., Sanabria-Diaz, G., Romero-Quintana, Y., Salazar-Guerra, I., Carballoso-Acosta, M., \& CaballeroMoreno, A. (2016). Functional connectivity and quantitative EEG in women with alcohol use disorders: A resting-state study. Brain Topography, 29(3), 368-381. doi:10.1007/ s10548-015-0467-x 
Houston, R. J., \& Ceballos, N. A. (2013). Human neurophysiology: EEG and quantitative EEG in addiction research. In P. Miller (Ed.), Biological research on addiction (Chapter 38, pp. 379-390). Amsterdam, The Netherlands: Elsevier Inc.

Huang, E. J., \& Reichardt, L. F. (2001). Neurotrophins: Roles in neuronal development and function. Annual Review of Neuroscience, 24(1), 677-736. doi:10.1146/annurev.neuro.24.1.677

Jung, T.-P., Makeig, S., Humphries, C., Lee, T.-W., Mckeown, M. J., Iragui, V., \& Sejnowski, T. J. (2000). Removing electroencephalographic artifacts by blind source separation. Psychophysiology, 37(2), 163-178. doi:10.1111/1469-8986. 3720163

Kamarajan, C., \& Porjesz, B. (2012). Brain waves in impulsivity spectrum disorders. In M. A. Cyders (Ed.), Psychology of impulsivity (pp. 20-93). Hauppauge, NY: Nova Science Publishers.

Kang, Y., \& Harris, L. (2000). Handedness and footedness in Korean college students. Brain and Cognition, 43(1-3), 268-274.

Kardos, Z., Tóth, B., Boha, R., File, B., \& Molnár, M. (2017). Age-dependent characteristics of feedback evaluation related to monetary gains and losses. International Journal of Psychophysiology, 122, 42-49. doi:10.1016/j.ijpsycho.2016. 05.007

Kim, A., Cha, J., Kwon, S., \& Lee, S. (2011). Construction and validation of Korean version of CPGI. Korean Journal of Psychology, 30(4), 1011-1038.

Knott, V., Cosgrove, M., Villeneuve, C., Fisher, D., Millar, A., \& McIntosh, J. (2008). EEG correlates of imagery-induced cigarette craving in male and female smokers. Addictive Behaviors, 33(4), 616-621. doi:10.1016/j.addbeh.2007.11.006

Lasa, L., Ayuso-Mateos, J., Vazquez-Barquero, J., DiezManrique, F., \& Dowrick, C. (2000). The use of the Beck Depression Inventory to screen for depression in the general population: A preliminary analysis. Journal of Affective Disorders, 57(1), 261-265. doi:10.1016/S0165-0327(99)00088-9

Lee, J. Y., Park, S. M., Kim, Y. J., Kim, D. J., Choi, S.-W., Kwon, J. S., \& Choi, J.-S. (2017). Resting-state EEG activity related to impulsivity in gambling disorder. Journal of Behavioral Addictions, 6(3), 387-395. doi:10.1556/2006.6.2017.055

Lee, K. W., Kim, S. Y., Chung, W., Hwang, G. S., Hwang, Y. W., \& Hwang, I. H. (2009). The validity and reliability of Korean version of Lubben Social Network Scale. Korean Journal of Family Medicine, 30(5), 352-358. doi:10.4082/kjfm.2009. 30.5.352

Lei, X., Wang, Y., Yuan, H., \& Mantini, D. (2014). Neuronal oscillations and functional interactions between resting state networks. Human Brain Mapping, 35(7), 3517-3528. doi:10.1002/hbm. 22418

Li, X., \& Wolf, M. E. (2015). Multiple faces of BDNF in cocaine addiction. Behavioural Brain Research, 279, 240-254. doi:10.1016/j.bbr.2014.11.018

Lorains, F. K., Cowlishaw, S., \& Thomas, S. A. (2011). Prevalence of comorbid disorders in problem and pathological gambling: Systematic review and meta-analysis of population surveys. Addiction, 106(3), 490-498. doi:10.1111/j.1360-0443.2010. 03300.x

Mantini, D., Perrucci, M. G., Del Gratta, C., Romani, G. L., \& Corbetta, M. (2007). Electrophysiological signatures of resting state networks in the human brain. Proceedings of the National Academy of Sciences of the United States of America, 104(32), 13170-13175. doi:10.1073/pnas.0700668104

Massar, S., Rossi, V., Schutter, D., \& Kenemans, J. (2012). Baseline EEG theta/beta ratio and punishment sensitivity as biomarkers for feedback-related negativity (FRN) and risktaking. Clinical Neurophysiology, 123(10), 1958-1965. doi:10.1016/j.clinph.2012.03.005

Park, S. M., Lee, J. Y., Kim, Y. J., Lee, J.-Y., Jung, H. Y., Sohn, B. K., Kim, D. J., \& Choi, J.-S. (2017). Neural connectivity in Internet gaming disorder and alcohol use disorder: A restingstate EEG coherence study. Scientific Reports, 7(1), 1333. doi:10.1038/s41598-017-01419-7

Pezawas, L., Verchinski, B. A., Mattay, V. S., Callicott, J. H., Kolachana, B. S., Straub, R. E., Egan, M. F., MeyerLindenberg, A., \& Weinberger, D. R. (2004). The brain-derived neurotrophic factor val66met polymorphism and variation in human cortical morphology. The Journal of Neuroscience, 24(45), 10099-10102. doi:10.1523/JNEUROSCI.2680-04.2004

Pu, L., Liu, Q.-S., \& Poo, M.-M. (2006). BDNF-dependent synaptic sensitization in midbrain dopamine neurons after cocaine withdrawal. Nature Neuroscience, 9(5), 605-607. doi:10.1038/nn1687

Quintero, G. C. (2017). A biopsychological review of gambling disorder. Neuropsychiatric Disease and Treatment, 13, 51-60. doi:10.2147/NDT.S118818

Rangaswamy, M., Porjesz, B., Chorlian, D. B., Wang, K., Jones, K. A., Bauer, L. O., Rohrbaugh, J., O'Connor, S. J., Kuperman, S., Reich, T., \& Begleiter, H. (2002). Beta power in the EEG of alcoholics. Biological Psychiatry, 52(8), 831-842. doi:10.1016/S0006-3223(02)01362-8

Son, K., Choi, J., Lee, J., Park, S., Lim, J., Lee, J., Kim, S., Oh, S., Kim, D., \& Kwon, J. (2015). Neurophysiological features of Internet gaming disorder and alcohol use disorder: A restingstate EEG study. Translational Psychiatry, 5(9), e628. doi:10.1038/tp.2015.124

Szeszko, P. R., Lipsky, R., Mentschel, C., Robinson, D., Gunduz-Bruce, H., Sevy, S., Ashtari, M., Napolitano, B., Bilder, R. M., Kane, J. M., Goldman, D., \& Malhotra, A. K. (2005). Brain-derived neurotrophic factor val66met polymorphism and volume of the hippocampal formation. Molecular Psychiatry, 10(7), 631-636. doi:10.1038/sj.mp.4001656

Tammela, L. I., Pääkkönen, A., Karhunen, L. J., Karhu, J., Uusitupa, M. I., \& Kuikka, J. T. (2010). Brain electrical activity during food presentation in obese binge-eating women. Clinical Physiology and Functional Imaging, 30(2), 135140. doi:10.1111/j.1475-097X.2009.00916.x

Terracciano, A., Piras, M. G., Lobina, M., Mulas, A., Meirelles, O., Sutin, A. R., Chan, W., Sanna, S., Uda, M., \& Crisponi, L. (2013). Genetics of serum BDNF: Meta-analysis of the Val66Met and genome-wide association study. The World Journal of Biological Psychiatry, 14(8), 583-589. doi:10.3109/15622975. 2011.616533

Thibodeau, R., Jorgensen, R. S., \& Kim, S. (2006). Depression, anxiety, and resting frontal EEG asymmetry: A meta-analytic review. Journal of Abnormal Psychology, 115(4), 715-729. doi:10.1037/0021-843X.115.4.715

Von Stein, A., \& Sarnthein, J. (2000). Different frequencies for different scales of cortical integration: From local gamma to 
long range alpha/theta synchronization. International Journal of Psychophysiology, 38(3), 301-313. doi:10.1016/S01678760(00)00172-0

Wölfling, K., Mörsen, C. P., Duven, E., Albrecht, U., Grüsser, S. M., \& Flor, H. (2011). To gamble or not to gamble: At risk for craving and relapse - Learned motivated attention in pathological gambling. Biological Psychology, 87(2), 275281. doi:10.1016/j.biopsycho.2011.03.010

World Medical Association. (1964). Declaration of Helsinki: Ethical Principles for Medical Research Involving Human Subjects. Helsinki, Finland: WMA General Assembly.
Yamada, K., \& Nabeshima, T. (2003). Brain-derived neurotrophic factor/TrkB signaling in memory processes. Journal of Pharmacological Sciences, 91(4), 267-270. doi:10.1254/ jphs.91.267

Zoon, H. F., Veth, C., Arns, M., Drinkenburg, W., Talloen, W., Peeters, P. J., \& Kenemans, J. (2013). EEG alpha power as an intermediate measure between brain-derived neurotrophic factor Val66Met and depression severity in patients with major depressive disorder. Journal of Clinical Neurophysiology, 30(3), 261-267. doi:10.1097/WNP.0b013 e3182933d6e 